\title{
Some Traits on the Outcome of the Treatment of Cervical Cancer in Tanzania: A Case Study of Ocean Road Cancer Institute (ORCI)
}

\author{
Leguma L. Bakari', Rajabu R. Akarro², Amina S. Msengwa ${ }^{2{ }^{*} 1}$ and Francis J. Sichona ${ }^{2}$ \\ ${ }^{1}$ Eastern Africa Statistical Centre, P.O.Box 35103 Dar es Salaam, Tanzania \\ ${ }^{2}$ University of Dar es Salaam, Department of Statistics, P.O. Box 35047, Dar es Salaam, Tanzania
}

\begin{abstract}
Background: In Tanzania, like in many other poor African countries, cervical cancer is a major problem facing women especially for those aged 30 years and above. This study aimed at constructing a statistical model to enable the prediction of the outcome of treatment for cervical cancer patients in Tanzania.

Methods: Data were collected retrospectively from patient's files with histological proven cervical cancer who were treated at Ocean Road Cancer Institute (ORCl) from year 2009 and followed up to year 2011. The factors considered are screening status, HIV status, disease stage, age, treatment type and the intent of the treatment. The study employed the Chi-square $\left(\chi_{2}\right)$ test and the logistic regression model for its analysis.

Results: The Chi-square $\left(\chi_{2}\right)$ test result showed that there was a significant relationship between outcome of treatment and the patient screening status, HIV status, disease stage and intent of treatment at $5 \%$ level of significance. On the other hand, the logistic regression results found patient disease stage and intent of the treatment to be statistically significant at 95 percent. Logistic regression results also showed that patients who attended ORCI when their disease at a late stage had an odds ratio of 0.128 less likely to have favorable outcome compared to those patients who attended $\mathrm{ORCl}$ when their disease stage was at early stages. The odds ratio for cervical cancer patients who received both treatment, radiotherapy, and chemotherapy was 2.643 more likely to have favorable outcomes

Conclusion: More emphasis and campaigns should be made in order to encourage women all over the country to attend cancer centers for screening and treatment at early stages or even before any symptoms for cervical cancer and other types of cancers.
\end{abstract}

Keywords: Tanzania, cervical cancer, women, age, logistic regression, ORCI

\section{Introduction}

Cancer is a generic term used to describe a group of over 100 diseases that occur when malignant forms of abnormal cell growth develop in one or more body organs (Bruni et al. 2015; WHO 2014; FWC 2013). Cervical cancer is much common in developing countries compared to developed countries (Bray et al. 2012). Cancer of the cervix is the most common cancer among women in sub-Saharan Africa, which alone had 22\% of all cervical cancer cases reported globally in 2010 (SALC 2012).

All women are at risk for cervical cancer, occurs most often in women over age 30 (Ashford and Collymore 2005; Senkomago 2017). The primary risk factor for developing cervical cancer is being infected with the Human Papilloma Virus (HPV) a common virus that is transmitted through sexual contact (SALC 2012; Reis et al. 2011). The other risk factors are smoking, HIV/AIDS, using birth control pills for a long time, etc. (Senkomago 2017; Malik 2005). According to SRADAH (2014), Tanzania was ranked as the 6th country in Africa with high incidence of cervical cancer, and also ranked as the $5^{\text {th }}$ country in Africa with a high cumulative risk of cervical cancer incidence. According to Ministry of Health and Social Welfare (2011), there were about 24 different type of cancers for women who attended $\mathrm{ORCl}$. There were about 16,246 cases for all cancers, and among them, 7,515 were cervical cancer cases equivalent to $46.3 \%$ of all cancer cases (MoHSW 2011). It indicates that nearly half of all women cancer cases were cervical cancer. In

\footnotetext{
$1 *$ Corresponding author amina.msengwa@yahoo.com
} 
Tanzania, about 7,304 new cervical cancer cases are diagnosed, and about 4,216 deaths occur annually, and as indicated by the estimate of the year 2012 (Bruni et al. 2015). Cervical cancer ranks as the first cause of female cancer in Tanzania, mostly to women aged 15 to 44 years in Tanzania (Bruni et al. 2015). This paper aims at constructing a statistical model depicting traits associated with the outcome of the treatment for cervical cancer patients in Tanzania so that remedial actions can be taken.

\section{Materials and Methods}

\section{Study Design}

A hospital-based retrospective study was conducted. This study used secondary data from the $\mathrm{ORCl}$. ORCI is the only specialized cancer hospital in Tanzania. The institution receives patients with histological diagnosis of cancer from all over the country and women with no symptoms who come for screening. The data were available at the medical records unit of Ocean Road Cancer Institute. Data were collected from patient's files with histological proven cervical cancer who were treated at ORCI from the year 2009 and followed up to the year 2011.

The study population was all women with cervical cancer in Tanzania, but the accessible population was all women referred to $\mathrm{ORCl}$ for treatment of cervical cancer and all cervical cancer women diagnosed at the ORCI cervical cancer screening clinic between 2009 and 2011. The dependent variables defined as "favorable" if the patient had been cured with no recurrence of disease after treatment and "unfavorable" if the patient had the recurrence of the disease, having persistence of symptoms or died after treatment. The outcome of the treatment status was obtained by tracking the medical records files available at the medical records unit of Ocean Road Cancer Institute. The risk factors for cervical cancer considered are factors that enhance woman chances of developing cervical cancer. This include infection with Human Papilloma Virus (HPV), smoking, immune suppression such as HIV/AIDS, early sexual activity, multiple sexual partners, family history of cervical cancer such as mother/father or sister/brother with a cervical cancer before, use of contraceptive pills, social-economic status.

\section{Logistic Regression Model}

Since the dependent variable is dichotomous a logistic regression model was used:

$\ln [p /(1-p)]=\alpha+\sum_{i=1}^{n} \beta_{i} x_{i} \quad$, Where $\mathrm{p}$ is the probability of a cervical cancer patient to have a

favorable outcome, $\alpha$ and $\beta_{i}$ are estimated regression coefficients, and $x_{i}$ are the explanatory variables:

$x_{1}$-patient screening status

$x_{3}$-patient disease stage

$x_{5}$-patient marital status

$x_{7}$-patient education level $x_{2}$-patient HIV status variable

$x_{4}$-patient intent of the treatment

$x_{6}$-patient zonal of residence

Before fitting the models, cross-tabulations of treatment outcome and the explanatory variables in categorized forms were done to assess the degree of association. The estimated model tells the amount of change in the log of odds of the outcome of the treatment that would be predicted by 1-unit change in a particular independent variable, holding other variables constant. The coefficients are in log-odds units and hence it is difficult to be interpreted. The easiest method to interpret them converts them to odds ratios by simply taking the exponent of the coefficient. 
Models were estimated using the SPSS software version 22. A stepwise procedure to arrive at the final model by a combination of forward selection and backward elimination was made. In addition to the $\mathrm{p}$ values, which are the output for each coefficient, an overall test of significance of each independent variable was done using a likelihood ratio test, which is mostly a chi-squared test on the difference in log-likelihoods. The test compares the fit to the data in a model where the variable considered is included and a model in which it is excluded, for details see Polissar \& Diehr (1982); Agresti (2007).

\section{Results}

\section{Demographic Characteristics}

Table 1 gives the profile of 216 patients who were historically proven and treated at $\mathrm{ORCI}$ from year 2009 and followed up to year 2011. Socio-demographic characteristics of the research respondents were zone, marital status, education, and age. These characteristics were crucial in determining the structure of analysis and enabled comparisons in the response categories.

Table 1: Distribution of Patients by Socio-Demographic Characteristics

\begin{tabular}{llcc}
\hline Variable & Category & Frequency & Percentage \\
\hline Age & $15-30$ & 6 & 2.8 \\
& $31-45$ & 87 & 40.3 \\
& $46-60$ & 66 & 30.5 \\
& $60+$ & 57 & 26.4 \\
\hline Marital Status & Total & Single & $\mathbf{1 0 0}$ \\
\hline & Married & $\mathbf{2 1 6}$ & 37.9 \\
& Divorced/Separated & 82 & 43.1 \\
& Widowed & 93 & 12.9 \\
& Cohabiting & 28 & 4.2 \\
& Total & 9 & 1.9 \\
& None & 9 & $\mathbf{1 0 0}$ \\
\hline Pducation & Secondary & 4 & 9.7 \\
& Above Sec & $\mathbf{2 1 6}$ & 45.4 \\
& Total & 21 & 38.4 \\
& Central & 98 & 6.5 \\
& Coastal & 83 & $\mathbf{1 0 0}$ \\
\hline Lone & Lake & 14 & 4.6 \\
& Sorthern & $\mathbf{2 1 6}$ & 60.2 \\
& Southern Highlands & 10 & 6.5 \\
& Total & 130 & 12.0 \\
& & 14 & 11.1 \\
& & 26 & 5.6 \\
& & 24 & $\mathbf{1 0 0}$ \\
\hline
\end{tabular}

Table 1 shows that $2.8 \%$ of the patients were aged between 15 and 30 years. Frequently most of the cervical cancer cases were coming to effect for those women aged 30 years and above; therefore, the young women in developing countries such as Tanzania were not performing regular checkups at cancer centers in order to discover the pre-cancerous lesions. On the other hand, $40.3 \%$ were aged between 31 and 45 years while 30.5\% were aged between 46 and 60 years and $26.4 \%$ were above 60 years. Approximately $37.9 \%$ of the patients were single (never married) while $43.1 \%$ were married. It also shows that $12.9 \%$ of the patients were divorced, $4.2 \%$ were widowed, and 1.9 cohabited. By combining those patients who were married, divorced, widowed and those who cohabited, about two-thirds (62.1\%) of the patients were active in sex relationship and therefore at risk of being infected with HPV and naturally at high risk of getting cervical cancer. Furthermore, $9.7 \%$ of the patients did not go to school at all, $45.4 \%$ attained primary education, and $38.4 \%$ attained secondary education and only $6.5 \%$ of the patients attained above secondary education. The majority of the patients were from Coastal Zone (60.2\%), $11.1 \%$ were 
from Southern Highlands Zone, $12 \%$ were from Northern Zone, $6.5 \%$ were from Lake Zone, $4.6 \%$ were from Central Zone, and 5.6\% were from Zanzibar. On the other hand, those patients who were from far regions such as Mara, Tabora, Mbeya, and Zanzibar were only $7 \%$.

\section{The relationship between treatment outcome and other variables}

A cross tabulation was performed to determine the association between the outcome of the treatment and screening status, disease stage, HIV status, treatment type, age and intent of the treatment.

Table 2: Associations between Treatment Outcome and Independent Factors

\begin{tabular}{|c|c|c|c|c|c|c|c|}
\hline \multirow[t]{3}{*}{$\begin{array}{l}\text { Responses } \\
\text { variables }\end{array}$} & \multirow[t]{3}{*}{ independent } & \multicolumn{3}{|c|}{$\begin{array}{l}\text { The response of Outcome of the } \\
\text { treatment }\end{array}$} & \multirow[t]{3}{*}{$x^{2}$} & \multirow[t]{3}{*}{$\mathbf{P}$} & \multirow[t]{3}{*}{$\begin{array}{l}\text { Odds } \\
\text { ratio }\end{array}$} \\
\hline & & Unfav & Fav & Total & & & \\
\hline & & 81 & 135 & 216 & & & \\
\hline \multirow[t]{2}{*}{ Screening status } & Not screened & $67(48.0)$ & $61(80.0)$ & 128 & 29.346 & 0.000 & 5.806 \\
\hline & Screened & $14(33.0)$ & $74(55.0)$ & 88 & & & \\
\hline \multirow[t]{2}{*}{ HIV status } & Negative & $40(50.6)$ & $95(84.4)$ & 135 & $9 \cdot 514$ & 0.002 & 0.411 \\
\hline & Positive & $41(30.4)$ & $40(50.6)$ & 81 & & & \\
\hline \multirow[t]{2}{*}{ Disease stage } & Early & $17(41.3)$ & $93(68.7)$ & 110 & 46.48 & 0.000 & 0.120 \\
\hline & Late & $64(39.7)$ & $42(66.3)$ & 106 & & & \\
\hline \multirow[t]{2}{*}{ Treatment type } & Radi only & $76(71.6)$ & $115(119.4)$ & 191 & 3.694 & 0.055 & 2.643 \\
\hline & Radi\&chem & $5(09.4)$ & $20(15.6)$ & 25 & & & \\
\hline \multirow[t]{2}{*}{ Age } & Above 40 & $60(59.3)$ & $98(98.7)$ & 158 & 0.057 & 0.812 & 1.079 \\
\hline & 40 and below & $21(21.7)$ & $37(36.3)$ & 58 & & & \\
\hline \multirow{2}{*}{$\begin{array}{l}\text { The intent of the } \\
\text { treatment }\end{array}$} & Curative & $22(40.9)$ & $87(68.1)$ & 109 & 28.152 & 0.000 & 0.206 \\
\hline & Palliative & $59(40.1)$ & $48(66.9)$ & 107 & & & \\
\hline
\end{tabular}

Cross-tabulation results between the outcome of the treatment and the independent variables are shown in Table 2. The Pearson chi-square results show that the age of the patient and the treatment type was insignificantly associated with the outcome of the treatment at $5 \%$ level. According to the patients' information obtained, the patients were either treated by radiotherapy or both radiotherapy and chemotherapy, although the variable was insignificant. Therefore, the type of treatments variable should not be ignored in consideration of determining the treatment outcome of the cervical cancer patient and more emphasis should also be made for the treatment type after undergoing the other essential procedures.

Screening status and the intent of treatment of the patients were significantly associated with the outcome of the treatment at $5 \%$ level. This reveals that more effort should be made by both Government and NGO's to initiate the cancer screening clinics at least to each regional hospital in Tanzania. The odds ratio for the cervical cancer patients who were screened was 5.806 times, more likely to have favorable outcome compared to the patients who were not screened. Table 2 also shows that the odds ratio for cervical cancer patients who attended ORCI when the disease was at a late stage was 0.12 times, less likely to have a favorable outcome of the treatment compared with those patients who attended at $\mathrm{ORCl}$ when the disease was at an early stage. This shows that cervical cancer patients admitted at an early stage had better outcomes than those patients admitted at a late stage. The odds ratio for cervical cancer patients who received both treatment, radiotherapy, and chemotherapy was 2.643 times, more likely to have favorable outcome compared to those patients who were treated by radiotherapy only. The odds ratio for the cervical cancer patients who were intended for palliative care were 0.206 times less likely to have favorable outcome compared to those patients who were intended for curative treatment.

Since all the independent variables had the odds ratio either below 1 or above 1 , except age of the patient variable which had the odds ration close to $1(O R=1.079)$, this indicated that there 
was an existence of associations between the outcome of the treatment and 5 out 6 of the independent variables (screening status, HIV status, disease stage, type of the treatment, and the intent of the treatment). The odds ratio results were also in agreement with the chi-square results by indicating the associations between the outcome of the treatments and screening status, HIV status, and disease stage. The odds ratio (OR) for screening status was 5.806 since the OR was above one, then it indicated that there was an existence of an association between screening status and the outcome of the treatment. Also, the odds ratio for HIV status was 0.411 , since the OR was below one, then it also indicates that there was an existence of an association between HIV status and the outcome of the treatment. Lastly, the odds ratio for disease stage was 0.12 since the OR was below one, then it also indicated that there was an existence of an association between disease stage and the outcome of the treatment.

\section{Results from the binary multiple logistic regression analysis}

Having identified significant relationships between dependent variables and independent variables, a multiple logistic regression analysis was carried out to see the effect of the independent variables on the dependent variable. All independent variables considered showed significant relationships at the $5 \%$ level with the dependent variable, and hence all were entered into the multiple logistic regression model. The results are presented in Table 3. Demographic variables (marital status, education level and zone of residence) were also included in the binary logistic regression analysis in order to determine if they had a significant effect on treatment outcome.

Table 3 shows that the value of the likelihood ratio (LR) chi-square test is 60.10 , and its degree of freedom is 7. The overall model p-value is 0.0000 . From Table 3 only two variables, i.e., disease stage and intent of the treatment are statistically significant. Disease stage has p-value $=0.000$ ( $p$-value $<0.05)$ and intent of treatment $p$-value $=0.006$ ( $p$-value<0.05). Other variables (screening status, HIV status, marital status, education level and zonal of residence) are not statistically significant.

Table 3: Estimation for Binary Multiple Logistic Regression Model

\begin{tabular}{llllll}
\hline Variable & B & OR & \multicolumn{2}{l}{$95 \%$ C.I for OR } & P-Value \\
\cline { 5 - 6 } & & & Lower & Upper & \\
\hline Screening Status & -0.58 & 0.560 & 0.155 & 2.029 & 0.377 \\
HIV Status & -0.431 & 0.650 & 0.332 & 1.272 & 0.209 \\
Disease Stage & -2.052 & 0.128 & 0.040 & 0.041 & $0.000^{*}$ \\
Intent of the Treatment & -1.011 & 0.364 & 0.178 & 0.745 & $0.006^{*}$ \\
Marital status & 0.051 & 1.052 & 0.689 & 1.606 & 0.814 \\
Zonal of residence & -0.088 & 0.916 & 0.719 & 1.166 & 0.475 \\
Education Status & 0.219 & 1.245 & 0.825 & 1.878 & 0.296 \\
Constant & 1.982 & 7.257 & & & 0.076 \\
\hline
\end{tabular}

Number of observation 216 Likelihood Ratio chi-square $(7)=60.10$ ( $p$-value $=0$ )

From Table 3 the odds ratio for patient disease stage variable is 0.128 . This indicates that the cervical cancer patients who attended $\mathrm{ORCl}$ at a late stage are 0.128 times less likely to obtain a favorable treatment outcome compared to the patient who reaches $\mathrm{ORCl}$ when the disease was at an early stage. Also, the result shows that the odds ratio for the patient intent of treatment is 0.364 . This indicates that the patients whose medicine can no longer work (palliative) are 0.364 times less likely to have a favorable outcome.

\section{Discussion}

The findings revealed that $56.9 \%$ of the patients attended to $\mathrm{ORCl}$ when they were over 45 years. Similar results were obtained by (Majinge 2011) who showed that $40.8 \%$ cervical cancer patients 
were aged between 36-50 years, 35.4\% were aged between 51-65 years and those who were above 66 years was 15.9\%. This indicates that cervical cancer in developing countries like Tanzania is usually detected at late ages and it is the major reason that, most of the cervical cancer cases are presented at late stages when it has a small chance of being treated. Although it is expensive for developing countries to introduce the prevention methods for cervical cancer like HPV vaccine for HPV 16, HPV 18, HPV 6 and HPV 11 which is proved to be effective (Munoz et al. 2004; Malik 2005; Schiffman et al. 2007; Kerr and Fiander 2009), it is highly recommended in order to reduce the effect of cervical cancer to Tanzanian women.

According to Centers for Disease Control \& Prevention FDA (2010), the HPV vaccine is for young women aged between 9-13 years old who are still not active in sex relationship. Since age is a crucial factor for controlling cervical cancer, therefore more sensitization on cervical cancer screening and HPV testis needed especially in developing countries and particularly Tanzania. These results are in agreement with (Sherris et al. 2009) who found that in low resource settings like Tanzania, the optimal age group for cervical cancer screening to achieve the most significant public health impact was 30-39 years old. The results show that age is an important factor for controlling the spread of cervical cancer.

The findings revealed that more than $50 \%$ of the patients were still not able to reach secondary education. The results differ with (Majinge 2011) who found that $61.3 \%$ they did not obtain a formal education, $33.7 \%$ reached primary education and only $5 \%$ were above primary education. We believed that the more a person is educated, the better he/she would readily understand the surrounding environment by learning. Also, in case of the cervical cancer problem, we expected the patient who spends more years in school then she could learn easily the methods of how to protect herself from being infected with cervical cancer. The two results show that there was an improvement in education, but still, more effort is still required since there were about $10 \%$ of the patients who were still ignorant, and in this era of science and technology even primary education was not enough for once to cope up with the developed countries. In comparisons with (Majinge 2011) results about $2.7 \%$ of cervical cancer patients were single, $65.2 \%$ were married, and those who were divorced and widowed were about $31.4 \%$, which indicated about $96.6 \%$ of the patients were once sexually active. This indicates that more attention should be paid to methods of detecting HPV such as HPV RNA and HPV DNA testing.

The Pearson chi-square results show that the age of the patient was insignificantly associated with the outcome of the treatment. This result differs with (Mosha et al. 2009) who found the age of the patient to be significant at $5 \%$ with p-value $=0.013$. On the other hand, the HIV status was significantly associated with the outcome of the treatment at $5 \%$ level with pvalue $=0.002$. Similar results were obtained by (Mosha et al. 2009) who found that HIV status was significant at $5 \%$ with p-value $=0.001$. This shows that HIV status is an important factor to be observed in order to determine the outcome of the treatment of cervical cancer patients. Furthermore, the disease stage of the patient was also significantly associated with the outcome of the treatment at $5 \%$ level with p-value $=0.000$. This is in agreement with (Mosha et al. 2009) who found that cervical cancer disease stage was significant at $5 \%$ level with $p$-value $=0.001$. This shows that a stage of disease for cervical cancer patients should be strongly considered when it comes to the outcome of the treatment of the cervical cancer patients. They also showed that the development of cervical cancer was much faster in HIV positive women than those who were HIV negative.

As it has already been noted that, the primary risk factor for developing cervical cancer is being infected by the human papillomavirus (SALC 2012). In comparisons with (Majinge 2011) results about $2.7 \%$ of cervical cancer patients were single, $65.2 \%$ were married, and those who were divorced and widowed were about $31.4 \%$, which indicated about $96.6 \%$ of the patients were once sexually active. This indicates that more attention should be paid to methods of detecting HPV such as HPV RNA and HPV DNA testing. 
Similar results were also obtained by (Mosha. et al. 2009) who found that patients with cervical cancer known to be HIV sero-positive were more likely to die or be discharged home for palliative care( unfavorable outcome).These results are also consistent with (Mosha et al. 2009) results which also found that cervical cancer patients with stage I/II (early stage) were lower in percentage than those in stage III/IV (late stage). As it has already been noted, the primary risk factor for developing cervical cancer is being infected by the human papilloma virus (Watson et al. 2008; Jayaprakash et al. 2011). Therefore, the government through the Ministry of Health and Social Welfare can facilitate the establishment of HPV vaccine for the young women (aged between 9-13years) who are still inactive in sex relationship across the country. This will help to reduce the number of women with cervical cancer problems when they become active in sex relationship. This year, 2018 the government of Tanzania has been sensitizing young women to undergo cervical tests in the whole country. The researchers commend the government of Tanzania for initiating a screening program as suggested by the study.

The findings revealed that more than $50 \%$ of the patients were from the Coastal Zone. The Coastal Zone includes the regions of Dar es Salaam, Pwani, Morogoro, Mtwara, and Lindi, the main reason here why most of the patients were from coastal regions is that the $\mathrm{ORCl}$ is located in coastal Zone in Dar es Salaam region. The other regions of this zone are all close to Dar es Salaam which makes it cheaper to reach the ORCI with small transport cost. Similar results were obtained by (Mosha et al. 2009) who conducted a study on "Factors Associated with Management of Cervical Cancer Patients at KCMC Hospital" which revealed that $69 \%$ of the cases of admitted cervical cancer patients were residents of Kilimanjaro region. Others were from nearby regions such as Arusha and Manyara were $16 \%$, Singida was $4.5 \%$, and Tanga was $3.5 \%$.

The study shows that there is a significant association between the outcome of the treatment and screening status, HIV status as well as the disease stage of the cervical cancer patients who attended ORCI. The odds ratio for the cervical cancer patients who were HIV positive was 0.411 times, less likely to have a favorable outcome of the treatment compared to the patients who were HIV negative. These results are consistent with SRADAH (2014) results who found that HIV significantly increased the risk of persistence to HPV infections, which could lead to cervical cancer. The results suggest that the cervical cancer patients should undergo a screening test at least once since it is associated with the outcome of their treatment. Screening test is not only crucial for prevention but also a gateway to treatment. The results also showed that there is a significant relationship between HIV status and the outcome of the treatment. This suggests that special care should be provided to the cervical cancer patients who are HIV positive. Lastly, the disease stage of the patients seems to have an impact to the treatment of the cervical cancer patients, and therefore more emphasis should be taken so that the patients should attend cancer center when the disease stage is still at its infancy.

\section{Conclusion}

The results suggest that, the cervical cancer patients should undergo screening test at least once since it was associated with the outcome of their treatment. The results also showed that there was an association between HIV status and the poor outcome of the treatment of the patients. This suggests that special care should be provided to the cervical cancer patients who are HIV positive. Lastly, the disease stage of the patients seemed also to have an impact to the treatment of the cervical cancer patients and more emphasizes should be taken so that the patients should reach cancer centre when the disease stage is still at its infancy stage. This reveals that more effort should be made by both Government and NGO's to initiate the cancer screening clinics at least to each regional hospital in Tanzania. 
Ethical Considerations: Permission to use data was obtained from the Ethical Clearance committee and the Academic Unit of Ocean Road Cancer Institute.

Competing interests: The authors declare that they have no competing interests.

\section{References}

Agresti, A. (2007). An introduction to categorical data analysis. $2^{\text {nd }}$ edition, Wiley.

Ashford, L. \& Collymore, Y. 2005. Preventing Cervical Cancer Worldwide. Population Reference bureau. Policy brief. Available from http://www.prb.org/pdfo5/PreventCervCancerBrief_Eng.pdf.

Bray, F., Jemal, A., Grey, N., Ferlay, J. and Forman, D., (2012). Global Cancer Transitions According to the Human Development Index (2008-2030): A Population-Based Study. Lancet Oncology, 13, 790-801.

Bruni L, Barrionuevo-Rosas L, Albero G, Aldea M, Serrano B, Valencia S, Brotons M, Mena M, CosanR, Muñoz J, Bosch FX, de Sanjosé S, Castellsagué X. (2015). Human Papillomavirus and Related Diseases in Tanzania. ICO Information Centre on HPV and Cancer. Summary Report 2014-12-18.

Centers for Disease Control \& Prevention FDA licensure of bivalent human papillomavirus vaccine (HPV2, Cervarix) for use in females and updated HPV vaccination recommendations from the Advisory Committee on Immunization Practices (ACIP) MMWR Morbidity and Mortality. Weekly Report. (2010);59(20):626.

Foundation for Women's Cancer (FWC). (2013). Understanding Cervical Cancer. Chicago, IL 60606.

Jayaprakash V., Reid M., Hatton E., Merzianu M., Rigual N., Marshall J. Human papillomavirus types 16 and 18 in epithelial dysplasia of oral cavity and oropharynx: a meta-analysis, 1985-2010. Oral Oncol. (2011); 47(11):1048-1054.

Kerr DJ and Fiander AN. (2009). Towards Prevention of Cervical Cancer in Africa. Afrox.

Majinge PMC. (2011). Outcome of the treatment of the Cervical Cancer Patients at Ocean Road Cancer Institute. Theses and Dissertations, MUHAS.

Malik Al. (2005). The Role of Human Papilloma Virus (HPV) in the aetiology of Cervical Cancer. Department of Surgery, Royal Free and University College Medical School, Rowland Hill, London, UK.

Ministry of Health and Social welfare, MoHSW. (2011). National Cancer Control Strategy. Dar es Salaam.

Mosha D, Mahande M, Ahaz J, Mosha M, Njau B, Kitali B, Obure J. (2009). Factors Associated with Management of Cervical Cancer Patients at KCMC Hospital, Tanzania: a retrospective crosssectional study. Tanzania Journal of Health Research, Vol. 11

Munoz N., Bosch F.X., Castellsagué X., Díaz M., de Sanjose S., Hammouda D. Against which human papillomavirus types shall we vaccinate and screen? (2004). The international perspective. International Journal of Cancer. 111(2):278-285.

Polissar, L. and Diehr, P. (1982): Regression analysis in health services research: the use of dummy variables. Medical care 20 (9), 959-966. 
Reis N, Beji NK and Kilic D. (2011). Risk Factors for Cervical Cancer: Results froma Hospital-Based CaseControl Study. International Journal of Hematology and Oncology. UHOD, pp3, vol 21.

Schiffman M., Castle P.E., Jeronimo J., Rodriguez A.C., Wacholder S. Human papillomavirus and cervical cancer. Lancet. (2007); 370 (9590):890-907.

Scorecard Research, Analysis \& Design by Africa Health (SRADAH) Human \& Social Development Information and Africa Coalition on Maternal Newborn. (2014). Africa Cervical Cancer MultiIndicator Incidence \& Mortality Scorecard.

Senkomago, V., Duran, D., Loharikar, A., Hyde, T. B., Markowitz, L. E., Unger, E. R and Saraiya, M. (2017). CDC Activities for Improving Implementation of Human Papillomavirus Vaccination, Cervical Cancer Screening, and Surveillance Worldwide. Emerging Infectious Diseases, 23(13). https://dx.doi.org/10.3201/eid2313.170603.

Sherris J, Wittet S, Kleine A, Sellors J, Luciani S, Sankaranarayanan R, Barone MA. (2009). Evidence-Based, Alternative Cervical Cancer Screening Approaches in Low Resource Settings. International Perspectives on Sexual and Reproductive Health. Vol 35 Iss.3: 147-152.

Southern Africa Litigation Centre, SALC. (2012). Improving Access to Cervical Cancer Services for Women in Southern Africa. Johannesburg.

Watson M., Saraiya M., Ahmed F., Cardinez C.J., Reichman M.E., Weir H.K. Using population-based cancer registry data to assess the burden of human papillomavirus-associated cancers in the United States: Overview of methods. Cancer. (2008); 113(S10):2841-2854.

World Health Organization (WHO). Human papillomavirus vaccines: WHO position paper, (2014). Weekly Epidemiology Rec. 2014; 89(43):465-92. Available from http://www .who.int/wer/2014/wer8943.pdf. 